\section{Esophageal tumor diagnosed by capsule endoscopy}

A 74-year-old man was referred to our unit with a 3-year history of typical heartburn symptoms. His heartburn had became refractory to treatment with proton pump inhibitors in the 3 weeks prior to presentation but it was not accompanied by dysphagia, anemia, hematemesis, or weight loss. He was recommended to have an upper gastrointestinal endoscopy but he refused to undergo this examination. After much discussion, he was offered the option of esophageal capsule endoscopy, with the proviso that if any abnormal finding was observed he would require standard upper gastrointestinal endoscopy. The patient swallowed the video capsule (PillCam ESO; Given Imaging, Yoqneam, Israel) without problems and the study lasted for 20 minutes and 23 seconds, during which time the capsule did not pass into stomach (although capsule excretion was confirmed later). The capsule study revealed a polypoid lesion in the mid-esophagus (Figure 1, Video 1), as well as mucosa distal to the polyp that resembled gastric mucosa and was consistent with a diagnosis of Barrett's esophagus.

To investigate the lesion seen in the capsule study further, the patient then underwent upper gastrointestinal endoscopy (Figure 2) with biopsy, and endoscopic ultrasonography. The ultrasound examination revealed a mass that arose from the mucosa and infiltrated to the muscular layer. One rounded, echo-poor lymph node, $7 \mathrm{~mm}$ in diameter, was observed adjacent to the lesion, so a TNM stage of T2 N1 was established. Histological studies showed pleomorphic cells with large nuclei forming nests with infiltrative borders and necrotic areas. The absence of corneal pearls led to the diagnosis of undifferentiated esophageal carcinoma.

Preliminary results seem to demonstrate that esophageal capsule endoscopy has a high diagnostic yield in the detection of Barrett's esophagus and esophageal varices $[1,2]$. However, to our knowledge, this is the first reported case of an esoph-

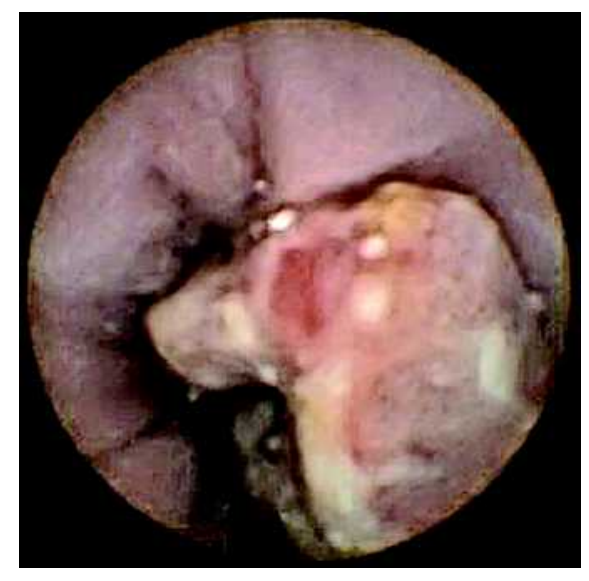

Figure 1 Esophageal capsule endoscopy revealed a polypoid lesion with a prominent area of nonbleeding ulceration at its apex (see also Video 1).

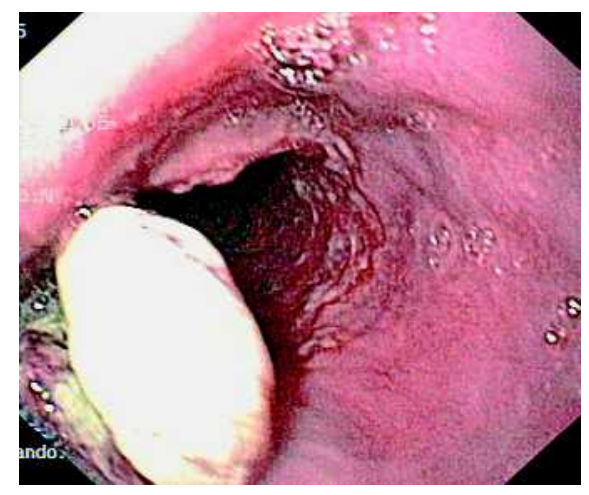

Figure 2 Conventional endoscopy confirmed the presence of the polypoid lesion.

ageal cancer diagnosed by esophageal capsule endoscopy, highlighting the potential usefulness of this technique for the detection of tumors. Nevertheless, the role of esophageal capsule endoscopy in screening for esophageal cancer must be assessed by well-designed, cost-effective studies.

\section{Video}

online content including video sequences viewable at: www.thieme-connect.de/ejournals/abstract/ endoscopy/doi/10.1055/s-2006-925192
Endoscopy_UCTN_Code_CCL_1AB_2AC_3AB

Endoscopy_UCTN_Code_CCL_1AB_2AH_3AB

A. Sánchez-Yagüe1, A. Caunedo-Álvarez', R. Romero-Castro ${ }^{1}$, J. Romero-Vázquez ${ }^{1}$, A. Antúnez-Infante ${ }^{2}$, F. Pellicer-Bautista $^{1}$, J. M. Herrerías-Gutiérrez ${ }^{1}$

${ }^{1}$ Department of Gastroenterology, Virgen Macarena University Hospital, Seville, Spain

${ }^{2}$ Department of Pathology, Virgen Macarena University Hospital, Seville, Spain.

\section{References}

${ }^{1}$ Eliakim R, Yassin K, Shlomi I et al. A novel diagnostic tool for detecting oesophageal pathology: the PillCam oesophageal videocapsule. Aliment Pharmacol Ther 2004; $20: 1$ - 7

2 Eisen G, De Franchis R, Eliakim R. Evaluation of esophageal varices by PillCam Eso as compared to upper endoscopy. Proceedings of the 4th International Conference on Capsule Endoscopy; 2005 March 7-8. Miami Beach, USA: 2005: 199

\section{Corresponding author}

\section{J. M. Herrerías Gutiérrez, Ph.D.}

Gastroenterology Department

8th Floor

Virgen Macarena University Hospital

Avda. Dr. Fedriani, 3

Sevilla 41009

Spain

Fax: $\quad$ +34955008805

E-mail: jmhg@us.es 